\title{
Chemical Structure of the Water-Insoluble Polysaccharide Isolated from the Fruiting Body of Ganoderma lucidum
}

\author{
Jinghua Chen, Jinping Zhou, Lina ZHANG, \\ Yo NAKAmura, ${ }^{*}$ and Takashi NorisuYE* \\ Department of Chemistry, Wuhan University, Wuhan 430072, People's Republic of China \\ * Department of Macromolecular Science, Osaka University, \\ Toyonaka, Osaka 560-0043, Japan
}

(Received May 6, 1998)

\begin{abstract}
Water-soluble and water-insoluble polysaccharide fractions were isolated from fruiting bodies of Ganoderma lucidum (rheishi) by extraction with phosphate buffer and $1 \mathrm{~N}$ sodium hydroxide solutions at different temperatures and investigated by high-performance liquid chromatography (HPLC), one- and two-dimensional ${ }^{1} \mathrm{H}$ and ${ }^{13} \mathrm{C} \mathrm{NMR}$, infrared (IR) spectroscopy, and other techniques. This work was prompted by Sone et al.'s earlier conclusion that similarly extracted, water-insoluble polysaccharides are $(1 \rightarrow 3)$ - $\beta$-D-glucans with very low degrees of branching though hardly hydrolyzed by the enzyme exo-( $1 \rightarrow 3)$ - $\beta$-D-glucanase of Basidiomycete QM 806. HPLC on the acid hydrolysates from our extracts showed that the water-soluble fractions were minor constituents and mostly heteropolysaccharides, while the water-insoluble ones (i.e., the alkali-extracts at 25 and $65^{\circ} \mathrm{C}$ ) were essentially pure glucans. It was found from NMR and viscosity measurements in dimethylsulfoxide containing $0.25 \mathrm{M} \mathrm{LiCl}$ that these glucans consist of linearly linked $(1 \rightarrow 3)$ - $\alpha$-D-glucose residues and differ only in molecular weight. The $(1 \rightarrow 3)$ - $\alpha$-structure of the water-insoluble fractions was confirmed by IR and optical rotation and also by comparison with the NMR spectra taken for standard $(1 \rightarrow 3)$ - $\beta$-D-glucan and $(1 \rightarrow 3)$ - $\alpha$-D-glucan samples.

KEY WORDS Polysaccharide / Ganoderma lucidum (Rheishi)/ Chemical Structure / Nuclear Magnetic Resonance / $(1 \rightarrow 3)-\alpha-$ D-Glucan / $(1 \rightarrow 3)-\beta$-D-Glucan /
\end{abstract}

The fruiting body of Ganoderma lucidum, called lingzhi in China and rheishi in Japan, has long been used as Chinese medicine in Eastern countries. Its medicinal effects on cancer, hypertension, hepatitis, and hypercholesterolemia have been demonstrated by pharmacological studies in the last two or three decades. ${ }^{1}$ On the other hand, physico-chemical properties of its constituent polysaccharides are as yet left almost unexplored. Although a few groups ${ }^{2-5}$ studied the chemical structures of the major constituents in relation to antitumor activity against Sarcoma 180 implanted in mice, the proposed polysaccharide structures are not conclusive for reasons given below.

Mizuno et al. ${ }^{2,4}$ extracted water-soluble and waterinsoluble polysaccharides from the fruiting body of Ganoderma lucidum, and concluded from bioassay and various chemical analyses that a water-soluble $(1 \rightarrow 3)$ - $\beta$ D-glucan with $(1 \rightarrow 6)$ - $\beta$-linked glucose side chains and some acidic heteropolysaccharides are responsible for the antitumor activity of rheishi. Sone et al. ${ }^{5}$ drew the following conclusions from similar but more detailed analyses. 1. The major constituents of the extracts from rheishi are water-insoluble $(1 \rightarrow 3)$ - $\beta$-D-glucans with very low degrees of branching. 2. These glucans have significantly lower antitumor activity than water-soluble $(1 \rightarrow 3)$ - $\beta$-D-glucans with higher degrees of $(1 \rightarrow 6)-\beta$ branching (DB). 3. Most water-soluble components of rheishi are heteropolysaccharides, water-soluble $(1 \rightarrow 3)$ $\beta$-D-glucans being minor constituents. Sone et al. remarked, however, that the water-insoluble $(1 \rightarrow 3)-\beta$-Dglucans are hardly hydrolyzed by the enzyme exo-(1 $\rightarrow 3)$ $\beta$-D-glucanase of Basidiomycete QM 806. This seems incompatible with the $(1 \rightarrow 3)-\beta$-structure with a low $\mathrm{DB}$, i.e., the above conclusion 1. Further, the two conclusions 1 and 2 are not always consistent with each other, in that schizophyllan ${ }^{6}$ (water-soluble $(1 \rightarrow 3)-\beta$-D-glucan with $\mathrm{DB}=1 / 3)$ and curdlan ${ }^{7}$ (water-insoluble $(1 \rightarrow 3)$ - $\beta$ $\mathrm{D}$-glucan with $\mathrm{DB}=0$ ) are known to have equally high antitumor activities.

The present study was undertaken to check the chemical structures proposed by Sone et al. ${ }^{5}$ for major constituent polysaccharides from rheishi. We extracted polysaccharides from fruiting bodies of Ganoderma lucidum and investigated water-insoluble components, i.e., alkali-extracts, by high-performance liquid chromatography, NMR, optical rotation, infrared spectroscopy, and other techniques.

\section{EXPERIMENTAL}

\section{Isolation of Polysaccharides}

Fruiting bodies of Ganoderma lucidum $(2 \mathrm{~kg})$ cultivated in Northeastern China were dried, crushed, and washed with hot ethylacetate and methanol over $4 \mathrm{~h}$. They were immersed and homogenized in $90 \%$ aqueous ethanol overnight to remove soluble compounds of low molar mass.

Water-soluble polysaccharides were extracted stepwise from the fruiting bodies with a $0.2 \mathrm{M}$ phosphate buffer solution (PBS) of $\mathrm{pH}=7$ at 25,80 , and $120^{\circ} \mathrm{C}$. In each step, the PBS suspension was centrifuged, and to the supernatant was added a large quantity of ethanol (EtOH) to precipitate the polysaccharide. The precipitates were designated GL1, GL2, and GL3 in order of increasing extraction temperature. The residue obtained from the last PBS suspension was treated with $1 N$ aqueous $\mathrm{NaOH}$ at 25 and $65^{\circ} \mathrm{C}$ to extract waterinsoluble fractions GL4-1 and GL4-2, respectively; acetic acid (HOAc) was used to precipitate the polymers. These extraction procedures, similar to those reported by Sone et $a l .{ }^{5}$ for rheishi and by Wang et al. ${ }^{8}$ for Ganoderma tsugae, are shown in Figure 1, along with the amounts 


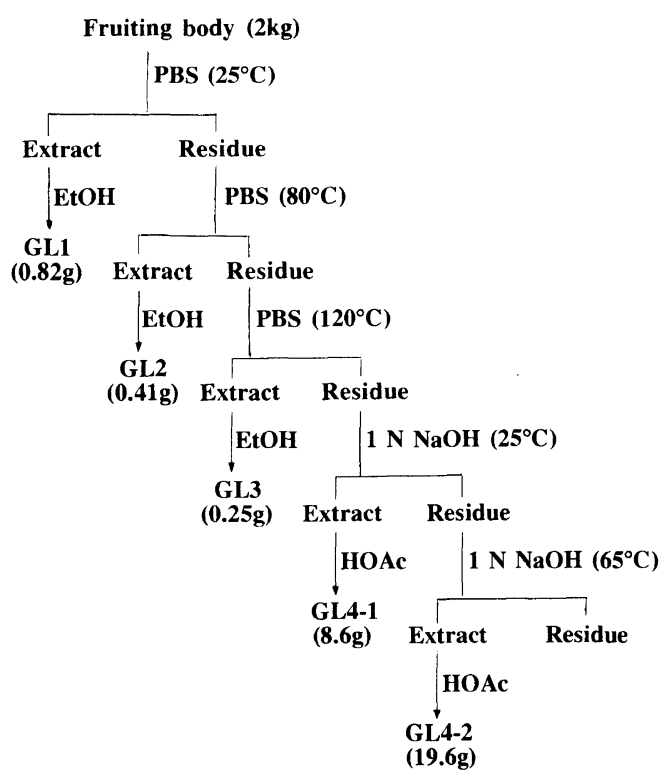

Figure 1. Extraction of polysaccharides from the fruiting body of Ganoderma lucidum. In each step, extraction was repeated three times with the indicated reagent of $5 \mathrm{~L}$ (i.e., $15 \mathrm{~L}$ in amount).

of the respective fractions. It can be seen that the alkali-extracts, i.e., the water-insoluble polysaccharides, were much richer than the water-soluble fractions. This is consistent with the finding of Sone et al. mentioned in the Introduction.

All fractions except GLl were treated by Sevag's procedure to remove proteins, and bleached by use of $20 \%$ aqueous hydrogen peroxide at $50^{\circ} \mathrm{C}$. They were lyophilized after their aqueous solutions or suspensions had been dialyzed against water to remove low molar mass compounds. Ultraviolet (UV) spectroscopy (Shimadzu UV-160A) in water or $1 N$ aqueous $\mathrm{NaOH}$ showed these fractions to have a single peak around $200 \mathrm{~nm}$; no UV absorption was detected at $280 \mathrm{~nm}$ (for proteins) and $600 \mathrm{~nm}$ (for pigments). Fraction GL1 was mostly polysaccharide-protein complexes and not examined in this work.

At an early stage of this work, we found from viscosity measurements that $1 N$ aqueous $\mathrm{NaOH}$ causes the waterinsoluble fractions GL4-1 and GL4-2 to degrade very slowly at room temperature. We therefore searched for an alternative solvent suitable for purification and viscometry. Solubility tests showed that the two fractions dissolve in dimethylsulfoxide (DMSO) containing an appropriate amount of lithium chloride $(\mathrm{LiCl})$ at room temperature and are stable in this mixed solvent system.

With $0.25 \mathrm{M} \mathrm{LiCl} / \mathrm{DMSO}$ (i.e., DMSO containing $0.25 \mathrm{M} \mathrm{LiCl}$ ) at $25^{\circ} \mathrm{C}$ as the solvent, fractions GL4-1 and GL4-2 were further purified by the addition of acetone, a precipitant. The resulting fractions were reprecipitated from $0.25 \mathrm{M} \mathrm{LiCl} / \mathrm{DMSO}$ solutions into $80 \%$ aqueous acetone, washed with $50 \%$ aqueous acetone three times and anhydrous acetone six times, and dried in vacuum for 7 days.

\section{HPLC}

Each fraction $\left(30 \mathrm{mg}\right.$ ) was dissolved in $4 \mathrm{~N} \mathrm{H}_{2} \mathrm{SO}_{4}$ $\left(5 \mathrm{~cm}^{3}\right)$ and hydrolyzed in a sealed tube at $100^{\circ} \mathrm{C}$ for $24 \mathrm{~h}$. The solution was neutralized by the addition of
$\mathrm{BaCO}_{3}$, and the $\mathrm{BaSO}_{4}$ produced was removed by centrifugation followed by filtration. After the solution had been concentrated to about $2 \mathrm{~cm}^{3}$, the sugar components were determined by high-performance liquid chromatography (HPLC) (Shimadzu LC-6A equipped with a refractive index detector and a $\mu$-Bondapak- $\mathrm{NH}_{2}$ column). A mixture of acetonitrile, water, and methanol ( $82: 12: 6$ by volume) was used for the mobile phase.

\section{Spectroscopy}

One- and two-dimensional ${ }^{1} \mathrm{H}$ and ${ }^{13} \mathrm{C}$ NMR measurements were made on a JEOL JNM-LA-500 spectrometer for the water-insoluble polysaccharide fractions GL4- 1 and GL4-2 in 0.25 M LiCl/DMSO- $d_{6}$ (deuterated DMSO) at $60^{\circ} \mathrm{C}$. The polymer concentration was adjusted to $5 \mathrm{wt} \%$ in all experiments.

A JASCO FT-IR 8300 spectrometer was used for infrared (IR) absorption spectroscopy. Test specimens were prepared by the $\mathrm{KBr}$-disk method.

Specific rotations $[\alpha]_{\mathrm{D}}$ at $589-\mathrm{nm}$ wavelength were determined on a JASCO ORD/UV5 spectropolarimeter with $0.5 \mathrm{~N}$ aqueous $\mathrm{NaOH}$ at $20^{\circ} \mathrm{C}$ as the solvent. A quartz cell of $10 \mathrm{~cm}$ in length was used, and the polymer mass concentration was adjusted to about $1 \times 10^{-2}$ $\mathrm{g} \mathrm{cm}^{-3}$.

\section{Viscometry and Light Scattering}

The intrinsic viscosity $[\eta]$ and Huggins' constant $k^{\prime}$ were determined for fractions GL4-1 and GL4-2 in $0.25 \mathrm{M} \mathrm{LiCl} / \mathrm{DMSO}$ at $25^{\circ} \mathrm{C}$ using a conventional viscometer of the Ubbelohde type. The density of this mixed solvent was determined to be $1.104 \mathrm{~g} \mathrm{~cm}^{-3}$ at $25^{\circ} \mathrm{C}$. DMSO was fractionally distilled under reduced pressure after being dehydrated with calcium hydride.

The higher molecular weight fraction GL4-2 (see below) in $0.25 \mathrm{M} \mathrm{LiCl} / \mathrm{DMSO}$ at $25^{\circ} \mathrm{C}$ was studied by light scattering with vertically polarized incident light of 436-nm wavelength to estimate its molecular weight, using a Fica 50 light scattering photometer (see ref 9 for the experimental procedures). When an analyzer was set in the horizontal direction, depolarized scattering was detected. Though the origin of the optical anisotropy was not clear, the weight-average molecular weight $M_{w}$ and the second virial coefficient $A_{2}$ were corrected for this effect by the conventional method ${ }^{10}$; the correction was about $10 \%$ for the former.

Test solutions were heated at $65^{\circ} \mathrm{C}$ for $20 \mathrm{~min}$ and then optically clarified by filtration through Teflon Millipore filters (at room temperature) followed by centrifugation at about $2.5 \times 10^{4}$ gravities for $2 \mathrm{~h}$. We note that virtually no change in $[\eta]$ at $25^{\circ} \mathrm{C}$ was observed before and after the heating.

The specific refractive index increment of fraction GL4-2 in $0.25 \mathrm{M} \mathrm{LiCl} / \mathrm{DMSO}$ at $25^{\circ} \mathrm{C}$ and $436 \mathrm{~nm}$ was determined to be $0.0580 \mathrm{~cm}^{3} \mathrm{~g}^{-1}$ for dialyzed solutions, using a modified Schulz-Cantow type differential refractometer. Dialysis was effected by the apparatus ${ }^{11}$ described elsewhere.

\section{RESULTS AND DISCUSSION}

\section{Carbohydrate Components}

HPLC analysis of the acid hydrolysates from the ex- 
tracts showed that the water-soluble fractions GL2 and GL3 are heteropolysaccharides containing D-glucose (only in GL2), D-galactose, D-mannose, D-xylose, L-fucose, and L-rhamnose, while the water-insoluble fractions GL4-1 and GL4-2 are almost pure glucans. D-glucuronic acid was detected in GL2 and GL3 by the carbazole reaction but neither in GL4-1 nor in GL4-2. These findings are quite similar to what was reported by Sone et al. $^{5}$

In contrast to our and Sone et al.'s findings, the "water-insoluble" fractions extracted by Mizuno et $\mathrm{al}^{4}{ }^{4}$ with $5 \%$ aqueous $\mathrm{NaOH}$ at $30^{\circ} \mathrm{C}$ were heteropolysaccharides and contained a substantial amount (about $10 \%$ ) of glucuronic acid. Though this inconsistency may come from a certain difference in the fruiting body and remains to be seen, the presence of the acid seems difficult to reconcile with the water-insolubility of the fractions.

Mizuno et al. $^{2}$ also isolated almost pure, water-soluble glucans. To see whether our water-soluble fraction GL2 contains a pure glucan, it was divided into five parts by gel permeation chromatography. Except for the third (central) fraction, the fractions were all heteropolysaccharides as reported by Sone et al. ${ }^{5}$ However, the central fraction was a pure glucan with a fairly large $[\eta]$ of $600 \mathrm{~cm}^{3} \mathrm{~g}^{-1}$ (without correction for shear rate) in $0.5 \mathrm{M}$ aqueous $\mathrm{NaCl}$ at $25^{\circ} \mathrm{C}$. Although such a large [ $\left.\eta\right]$ suggests that the polymer is semiflexible, no further characterization was feasible because of the very limited quantity.

\section{Structure of Water-Insoluble Polysaccharides}

One-dimensional ${ }^{1} \mathrm{H}$ and ${ }^{13} \mathrm{C}$ NMR spectra showed that the water-insoluble glucans GL4-1 and GL4-2 are chemically identical and have no side chain. The intrinsic viscosities for GL4-1 and GL4-2 in $0.25 \mathrm{M} \mathrm{LiCl} / \mathrm{DMSO}$ at $25^{\circ} \mathrm{C}$ were $37 \mathrm{~cm}^{3} \mathrm{~g}^{-1}\left(k^{\prime}=0.45\right)$ and $102 \mathrm{~cm}^{3} \mathrm{~g}^{-1}$ $\left(k^{\prime}=0.34\right)$, respectively. Thus these two fractions differ only in molecular weight. Our light scattering experiment yielded $M_{w}=1.95 \times 10^{5}$ and $A_{2}=1.33 \times 10^{-4} \mathrm{~mol}$ $\mathrm{cm}^{3} \mathrm{~g}^{-2}$ for the higher molecular weight fraction GL4-2.

Figure 2 shows the HMQC (heteronuclear multiquantum coherence) NMR spectrum (for GL4-1), which maps the correlation between the directly bonded $\mathrm{C}$ and $\mathrm{H}$ atoms, thus distinguishing the protons directly and indirectly linked to a $\mathrm{C}$ atom. The peaks for C-6 and $\mathrm{H}-6$ can readily be assigned, since only C-6 is directly bonded to two $\mathrm{H}$ atoms. Figure 3 shows the DQF (double-quantum filter) spectrum, which presents the correlation among the $\mathrm{H}$ atoms connected within three chemical bonds. The spectra in these two figures allow us to assign all the peaks for $\mathrm{H}, \mathrm{C}$, and $\mathrm{OH}$ with the aid of the already assigned C-6 and H-6 peaks. The results for the $\mathrm{H}$ and $\mathrm{C}$ atoms are summarized in Table I. The absence of OH-3 in the DQF spectrum (Figure 3) clearly shows the fraction to be a $(1 \rightarrow 3)$-D-glucan. The chemical shift of $5.1 \mathrm{ppm}$ for $\mathrm{H}-1$ is much closer to that expected for $\alpha$-glucose than for $\beta$-glucose (about $5.2 \mathrm{ppm}$ for $\alpha$-glucose and $4.6 \mathrm{ppm}$ for $\beta$-glucose in $\left.\mathrm{D}_{2} \mathrm{O}\right){ }^{12}$ This indicates that our fraction is not a $(1 \rightarrow 3)-\beta$-D-glucan but should be a $(1 \rightarrow 3)$ - $\alpha$-D-glucan.

To confirm this, we made additional NMR measurements on a commercially available curdlan sample (Wako Pure Chemical Ind.; Lot No. RSN 9512) under the same

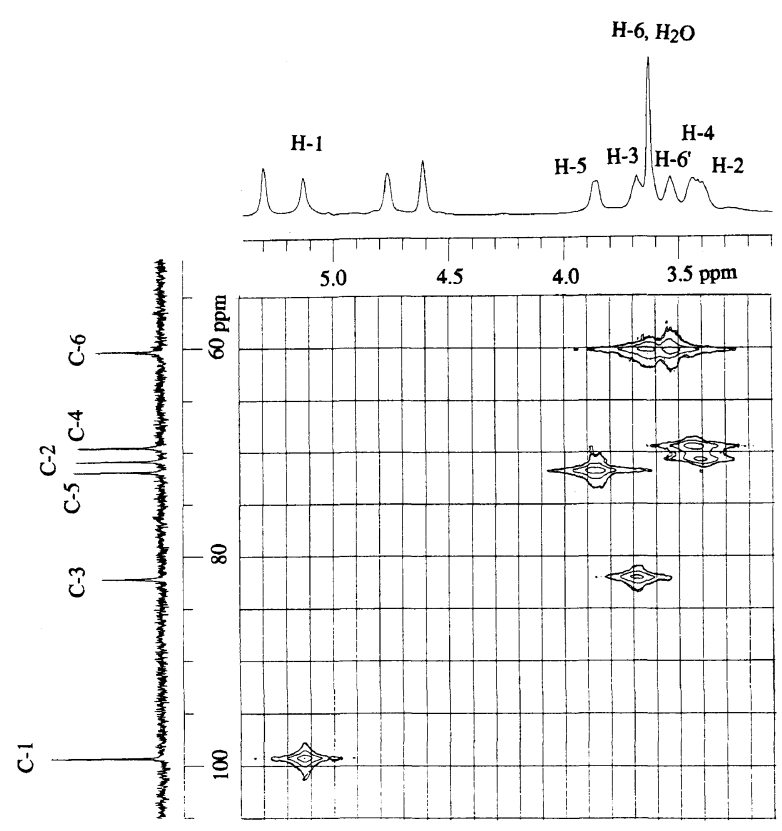

Figure 2. NMR spectrum (HMQC) for water-insoluble fraction GL4- 1 in $0.25 \mathrm{M} \mathrm{LiCl} / \mathrm{DMSO}-d_{6}$ at $60^{\circ} \mathrm{C}$.

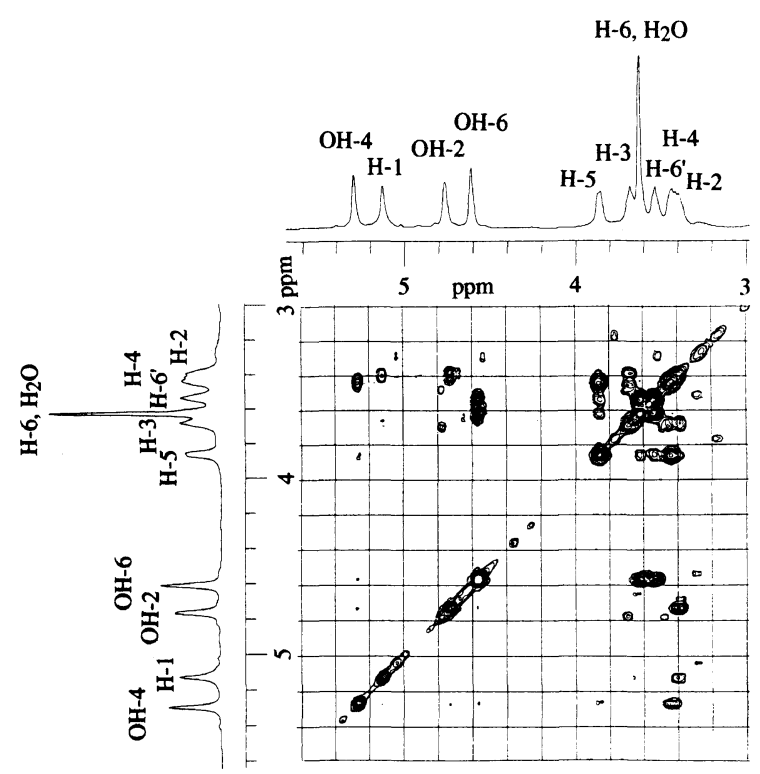

Figure 3. NMR spectrum (DQF) for water-insoluble fraction GL4-1 in $0.25 \mathrm{M} \mathrm{LiCl} / \mathrm{DMSO}-d_{6}$ at $60^{\circ} \mathrm{C}$.

conditions as for our water-insoluble fractions; note that curdlan also dissolves in mixtures of DMSO and $\mathrm{LiCl}^{13}$ The HMQC spectrum obtained is shown in Figure 4. The peaks have been assigned with the aid of the corresponding DQF spectrum (not shown here), but in actuality, the assignments of C-4 and C-5 have relied on the literature. ${ }^{14}$ Apparently, the HMQC spectrum for curdlan differs from the corresponding spectrum for GL4-1 in Figure 2. In particular, the difference in the chemical shift for $\mathrm{H}-1$ between the two glucans is distinct, substantiating that our polysaccharide cannot be a $(1 \rightarrow 3)-\beta$-D-glucan. The coupling constant ${ }^{3} J(\mathrm{H}-1, \mathrm{H}-2)$ for curdlan was estimated to be $7.5 \mathrm{~Hz}$, a value expected for $\beta$-glucose. ${ }^{12}$ That for our glucan could not be estimated since possibly the ${ }^{3} J(\mathrm{H}-1, \mathrm{H}-2)$ value expected for $\alpha$-glucose is as small as 3 to $4 \mathrm{~Hz}^{12}$ We further took 
Table I. Chemical shifts from ${ }^{13} \mathrm{C}$ and ${ }^{1} \mathrm{H}$ NMR spectra for the water-insoluble rheishi glucan in $0.25 \mathrm{M} \mathrm{LiCl} / \mathrm{DMSO}-d_{6}$ at $60^{\circ} \mathrm{C}$

\begin{tabular}{cccc}
\hline Assignment & Chemical shift $^{\text {a }}$ & Assignment & Chemical shift $^{\text {a }}$ \\
\hline C-1 & 99.3 & H-1 & 5.13 \\
C-2 & 70.9 & H-2 & 3.40 \\
C-3 & 82.2 & H-3 & 3.68 \\
C-4 & 69.5 & H-4 & 3.44 \\
C-5 & 71.9 & H-5 & 3.86 \\
C-6 & 60.4 & H-6 & 3.64 \\
& & H-6 & 3.53 \\
\hline
\end{tabular}

a in ppm.

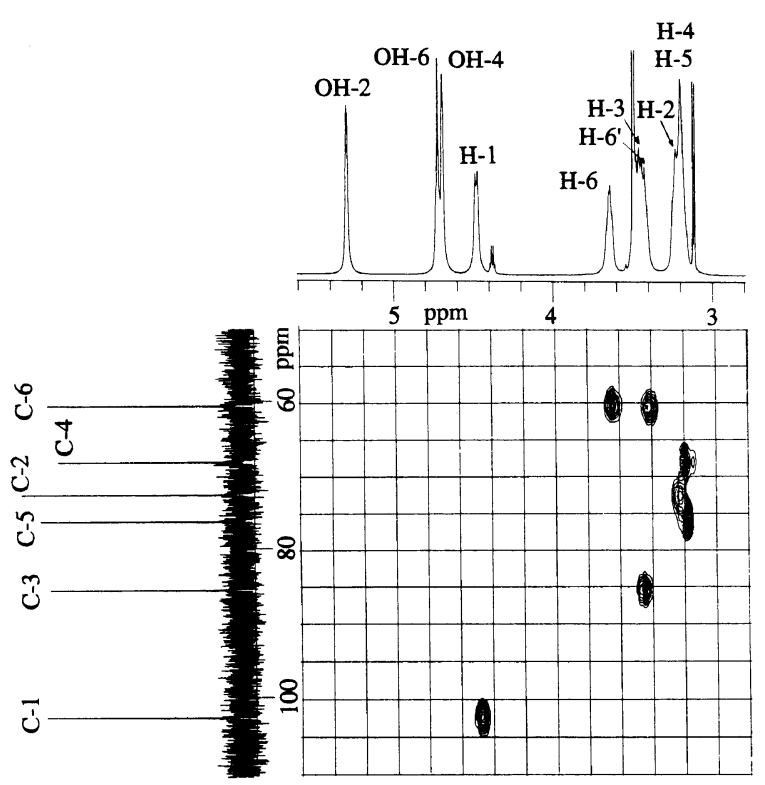

Figure 4. NMR spectrum (HMQC) for curdlan in $0.25 \mathrm{M} \mathrm{LiCl} /$ DMSO- $d_{6}$ at $60^{\circ} \mathrm{C}$.

NMR spectra for two enzymatically prepared $(1 \rightarrow 3)-\alpha-$ D-glucan samples furnished by Professor S. Kitamura of Kyoto Prefectural University; the values of $[\eta]$ for these samples in $0.25 \mathrm{M} \mathrm{LiCl} / \mathrm{DMSO}$ at $25^{\circ} \mathrm{C}$ were 30 and $106 \mathrm{~cm}^{3} \mathrm{~g}^{-1}$. The NMR spectra obtained were perfectly identical to those for the two fractions GL4-1 and GL4-2, thus confirming that the water-insoluble polysaccharide extracted from rheishi is a linear $(1 \rightarrow 3)$ - $\alpha$-D-glucan.

The values of $[\alpha]_{\mathrm{D}}$ for our glucan and curdlan in $0.5 \mathrm{~N}$ aqueous $\mathrm{NaOH}$ at $20^{\circ} \mathrm{C}$ were 170 and $-13 \mathrm{deg} \mathrm{cm}^{2} \mathrm{~g}^{-1}$, respectively. This characteristic difference also distinguishes between the $\alpha$-and $\beta$-type structures. Two points are to be noted here. First, the large positive $[\alpha]_{\mathrm{D}}$ value for our glucan is comparable to that $\left(195 \mathrm{deg} \mathrm{cm}^{2} \mathrm{~g}^{-1}\right.$ in $1 N$ aqueous $\mathrm{NaOH}$ ) reported for a $(1 \rightarrow 3)$ - $\alpha$-D-glucan from Agrocybe cylindracea by Kiho et al. ${ }^{15}$ Secondly, the normality $0.5 \mathrm{~N}$ of aqueous $\mathrm{NaOH}$ is high enough to break the possible helical conformation of curdlan ${ }^{16}$ and hence $[\alpha]_{D}$ of $-13 \mathrm{deg} \mathrm{cm}^{2} \mathrm{~g}^{-1}$ for that glucan may be considered to contain no substantial contribution from the helical structure.

Sone et al. $^{5}$ determined the chemical structures of water-insoluble fractions by gas-liquid chromatography, methylation, Smith degradation, and IR. Their results except from IR showed the backbone structures of the polysaccharide fractions to be composed of $(1 \rightarrow 3)$-D-

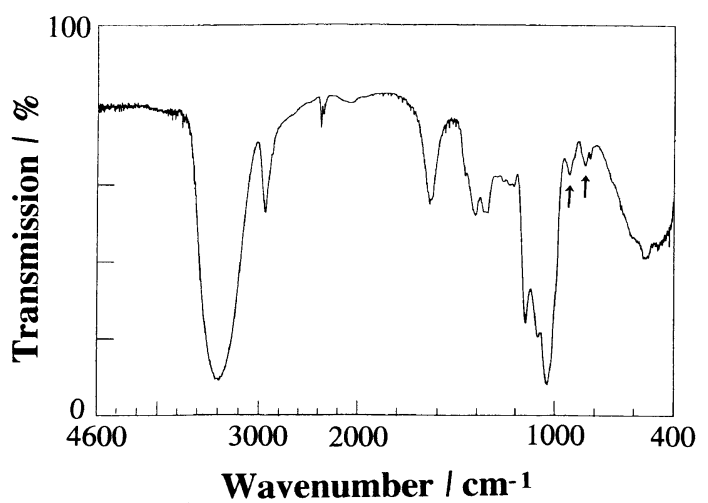

Figure 5. Infrared spectrum for fraction GL4-1. Arrows indicate absorption peaks at 849 and $927 \mathrm{~cm}^{-1}$.

glucose residues, as consistent with our NMR analysis. Their conclusion on the $\beta$-type came entirely from IR spectra reported to have an absorption peak at $890 \mathrm{~cm}^{-1}$ characteristic of a $(1 \rightarrow 3)$ - $\beta$-D-glucan. ${ }^{17,18}$

The IR spectrum for our glucan is shown in Figure 5. It has absorption peaks at 849 and $927 \mathrm{~cm}^{-1}$ characteristic of $\alpha$-D-glucans ${ }^{15,17,18}$ but no discernible peak at $890 \mathrm{~cm}^{-1}$. Thus, Sone et al.'s IR observation implies that their fractions contained $(1 \rightarrow 3)$ - $\beta$-D-glucans. However, since these glucans were hydrolyzed only to $2.6-5.5 \%$ by exo-(1 $\rightarrow 3)-\beta$-D-glucanase of Basidiomycete QM 806, their $\beta$ content must be such low.

\section{CONCLUDING REMARKS}

We showed by high performance liquid chromatography, NMR, optical rotation, and infrared spectroscopy that the water-insoluble polysaccharide extracted from the fruiting body of Ganoderma lucidum with $1 \mathrm{~N}$ aqueous $\mathrm{NaOH}$ is a linear $(1 \rightarrow 3)-\alpha$-D-glucan. This is consistent with the low degree of tumor inhibition found by Sone et al. ${ }^{5}$ for water-insoluble glucans, since the $(1 \rightarrow 3)$ - $\alpha$-glucan from Agrocybe cylindracea has a low antitumor activity against Sarcoma $180 .^{15}$ Thus, watersoluble fractions must be responsible for the antitumor activity of rheishi. Rheishi has other medicinal effects, ${ }^{1}$ so that it is intriguing to test the alkali-extract for those effects.

Except for the crystalline structure determined by $\mathrm{X}$-ray diffraction, ${ }^{19}$ little is known about the physicochemical properties of a linear $(1 \rightarrow 3)-\alpha$-D-glucan. This is probably because this type of glucan is seldom available though it is present in a number of fungi in crystalline form. ${ }^{20}$ The present study shows that almost pure, linear $(1 \rightarrow 3)$ - $\alpha$-D-glucan samples can be obtained from the fruiting body of Ganoderma lucidum as described here.

Acknowledgments. We are very grateful to Professor S. Kitamura of Kyoto Prefectural University for the gift of $(1 \rightarrow 3)$ - $\alpha$-D-glucan samples. We learned much about NMR of carbohydrates from Dr. Y. Suda of Osaka University, to whom many thanks are due. J. Chen gratefully acknowledges the Association of International Education, Japan, for the Short-Term Exchange Promotion Program, Peace and Friendship Scholarship, which enabled him to study at Osaka University for one year. L. Zhang also acknowledges with many thanks the 
National Natural Science Foundation of China for supporting this work.

\section{REFERENCES AND NOTES}

1. References can be found by on-line literature search. See also references cited in ref 2 .

2. T. Mizuno, N. Kato, A. Totsuka, K. Takenaka, K. Shinkai, and M. Shimizu, Nippon Nōgeikagaku Kaishi, 58, 871 (1984).

3. T. Miyazaki and M. Nishijima, Carbohydr. Res., 109, 290 (1982).

4. T. Mizuno, E. Suzuki, K. Maki, and H. Tamaki, Nippon Nōgeikagaku Kaishi, 59, 1143 (1985).

5. Y. Sone, R. Okuda, N. Wada, E. Kishida, and A. Misaki, Agric. Biol. Chem., 49, 2641 (1985).

6. K. Tabata, W. Ito, T. Kojima, S. Kawabata, and A. Misaki, Carbohydr. Res., 89, 121 (1981).

7. T. Sasaki, N. Abiko, Y. Sugino, and K. Nitta, Cancer Res., 38, 379 (1978).

8. G. Wang, J. Zhang, T. Mizuno, C. Zhuang, H. Ito, H. Mayuzumi, H. Okamoto, and J. Li, Biosci. Biotechnol. Biochem., 57, 894 (1993).
9. A. Tsuboi, M. Yamazaki, T. Norisuye, and A. Teramoto, Polym. J., 27, 1219 (1995).

10. H. Yamakawa, "Modern Theory of Polymer Solutions," Harper \& Row, New York, N.Y., 1971.

11. T. Sato, T. Norisuye, and H. Fujita, Macromolecules, 16, 185 (1983).

12. H. Friebolin, "Basic One- and Two-Dimensional NMR Spectroscopy," VCH, 1991.

13. M. Nakata, T. Kawaguchi, Y. Kodama, and A. Konno, Polymer, 39, 1475 (1998).

14. P. Colson, H. J. Jennings, and I. C. P. Smith, J. Amer. Chem. Soc., 96, 8081 (1974).

15. T. Kiho, I. Yoshida, K. Nagai, S. Ukai, and C. Hara, Carbohydr. Res., 189, 273 (1989).

16. K. Ogawa, T. Watanabe, J. Tsurugi, and S. Ono, Carbohydr. Res., 23, 399 (1972).

17. S. A. Barker, E. J. Bourne, and D. H. Whiffen, Methods Biochem. Anal., 3, 213 (1956).

18. A. J. Michell and G. Scurfield, Aust. J. Biol. Sci., 23, 345 (1970).

19. K. Ogawa, K. Okamura, and A. Sarko, Int. J. Biol. Macromol., 3, 31 (1981).

20. J. Jelsma and D. R. Kreger, Carbohydr. Res., 71, 51 (1979). 